\title{
Developing Effective and Efficient care pathways in chronic Pain: DEEP study protocol
}

\author{
Justin Durham ${ }^{1,2^{*}}$, Matthew Breckons ${ }^{1}$, Vera Araujo-Soares ${ }^{1}$, Catherine Exley ${ }^{1}$, Jimmy Steele ${ }^{1,2}$ and Luke Vale
}

\begin{abstract}
Background: Pain affecting the face or mouth and lasting longer than three months ("chronic orofacial pain", COFP) is relatively common in the UK. This study aims to describe and model current care pathways for COFP patients, identify areas where current pathways could be modified, and model whether these changes would improve outcomes for patients and use resources more efficiently.

Methods/Design: The study takes a prospective operations research approach. A cohort of primary and secondary care COFP patients $(n=240)$ will be recruited at differing stages of their care in order to follow and analyse their journey through care. The cohort will be followed for two years with data collected at baseline 6, 12, 18, and 24 months on: 1) experiences of the care pathway and its impacts; 2) quality of life; 3) pain; 4) use of health services and costs incurred; 5) illness perceptions. Qualitative in-depth interviews will be used to collect data on patient experiences from a purposive sub-sample of the total cohort $(n=30)$ at baseline, 12 and 24 months. Four separate appraisal groups (public, patient, clincian, service manager/commissioning) will then be given data from the pathway analysis and asked to determine their priority areas for change. The proposals from appraisal groups will inform an economic modelling exercise. Findings from the economic modelling will be presented as incremental costs, Quality Adjusted Life Years (QALYS), and the incremental cost per QALY gained. At the end of the modelling a series of recommendations for service change will be available for implementation or further trial if necessary.

Discussion: The recent white paper on health and the report from the NHS Forum identified chronic conditions as priority areas and whilst technology can improve outcomes, so can simple, appropriate and well-defined clinical care pathways. Understanding the opportunity cost related to care pathways benefits the wider NHS. This research develops a method to help design efficient systems built around one condition (COFP), but the principles should be applicable to a wide range of other chronic and long-term conditions.
\end{abstract}

Keywords: Orofacial pain, Health economics, Quality of life, Qualitative methods, Chronic pain, Care pathways

\section{Background}

Chronic pain is a distressing problem for patients and is difficult, and sometimes distressing, to manage for clinicians [1-4]. Chronic orofacial pain (COFP) affects a reported $13 \%$ of the UK population, and is particularly complex and distressing for patients [5-9]. Diagnosis and treatment for COFP conditions is slowly improving through the institution of new, targeted, diagnostic tools [10] and advances in genomics [11], but current care

\footnotetext{
* Correspondence: Justin.durham@ncl.ac.uk

${ }^{1}$ Institute of Health \& Society, Newcastle University, Baddiley-Clark Building, Richardson Road, Newcastle upon Tyne NE2 4AX, UK

${ }^{2}$ Centre for Oral Health Research (COHR), Newcastle University, Newcastle
} upon Tyne, UK

\section{Biomed Central}

(c) 2014 Durham et al.; licensee BioMed Central Ltd. This is an Open Access article distributed under the terms of the Creative Commons Attribution License (http://creativecommons.org/licenses/by/2.0), which permits unrestricted use, distribution, and reproduction in any medium, provided the original work is properly cited. The Creative Commons Public Domain Dedication waiver (http://creativecommons.org/publicdomain/zero/1.0/) applies to the data made available in this article, unless otherwise stated. pathways do not seem to maximise therapeutic potential and paradoxically may worsen COFP $[6,12]$.

COFP patients are known to use more healthcare resource compared to other dental patients [13-17], but what is unknown is why, or where, this utilisation occurs and how effective it is. Previous research $[1,6,12,13]$ seems to suggest that a large proportion of this resource utilisation may occur as a result of inadequate care pathways for patients with COFP: cyclical referrals accompanied by multiple and unnecessary consultations which often only serve to increase confusion and sometimes worsen the patient's complaint [6]. This is a costly process for both the patient and the health service and therefore in addition to delivering more accurate diagnoses and treatment there is an urgent 
need to understand how and where services can be streamlined in order to get patients to the most appropriate care effectively and efficiently.

A simplistic unidimensional assessment of the costs of care pathways is insufficient to capture the biopsychosocial dynamic relationship of COFP and the care received [18]. A "whole systems perspective" [19] is required in order to assess current care pathways and produce patient centred services "designed around patient's needs" [20]. This will help achieve one of the recent recommendations of the recent national pain audit in the UK, which is to "research... optimal models of care for people with chronic pain, including economic modelling" [21]. Without identifying where the negative economic, biomedical, and psychosocial impacts exist on the current care pathway from both the consumer and the providers' perspectives, it is impossible to model new pathways that provide appropriate care in a patient-centred, efficient, efficacious and expedient manner.

\section{Aims and objectives}

This study will describe and model current care pathways for COFP patients, identify areas where the current pathways could be modified, and model the estimated impact of change to determine what changes would improve outcomes for patients and use resources more efficiently.

Specifically it will:

\section{Phase 1}

i) Develop a map of COFP patients' journeys through care and understand their experiences of the care pathway using qualitative in-depth interviews.

ii) Identify the impacts of the various stages of care pathways on: individual's pain (West Haven Yale Multidimensional Pain Inventory, WYMPI [22]; Graded Chronic Pain Scale, GCPS [23]); quality of life, (EQ-5D) and the value that patients attach to the various stages of their care pathway [24]; use of health service and patient costs (use of health service and patient costs questionnaire [25]); illness perceptions (Revised illness perceptions questionnaire, IPQ-R [26]).

\section{Phase 2}

iii) Develop a model based upon the care pathways reflecting key events (e.g. referrals, use of services, impact on pain and daily living) and use this model to estimate the cost and outcomes (e.g. level of pain, quality of life).

iv) Use the data gathered from objectives i to iii and work with stakeholders, to identify priority areas where the current pathway might be changed and model the impact of the potential changes on costs, outcomes and cost-effectiveness of care for COFP.

v) Use the results of (i-iv) above develop recommendations for practice and future research

\section{Methods/Design}

We propose to capture data across the journey through care for COFP patients. This journey will start with the experience of pain and the individual will seek help from the health service, most frequently through primary care, but not exclusively so. The care journey may, for a minority, be a short one, but the current literature suggests that multiple healthcare providers may be consulted about the condition and the journey may not be as linear as depicted by Figure $1[6,12,13,21]$. If this were the case it would mean that to capture the entirety of some individuals' care pathway(s) might take several years, which is impractical for a number of reasons. This study proposes to solve this problem by purposively sampling individuals suffering from COFP along the continuum of care from initial experience of pain through to a final outcome which may be successful treatment, or the acknowledgement no more can be done (Figure 1). In this manner we will be able to capture data from all aspects of the care journey and pathway for two years, which will result in some individuals describing whole journeys (Figure 1 - dark plus light grey boxes) and some describing particular aspects or points of the care pathway (Figure 1 - dark grey boxes).

This data collection across the continuum will allow a complete picture of the possible care pathways for COFP patients to be built. Data collection will be accomplished through qualitative and quantitative methods with the qualitative data helping explain any apparent relationships in the quantitative data.

\section{Phase 1 - Recruitment, observation, recording, and mapping the current pathway(s) Methods}

Patients will be recruited from Primary and Secondary care in the North East of England. Primary care recruitment will take place from 25 medical practices and 10 dental practices from diverse socioeconomic areas (Figure 2). Secondary care patients will be recruited from a variety of clinics in the local dental and medical hospitals: neurology, oral and maxillofacial surgery, dental emergency clinic, oral medicine, and restorative dentistry.

Using a total sample size of 200, with a Type I error of $5 \%$, we can, with $80 \%$ power, detect an effect size of 0.4 within our data (Two-tailed). This represents a moderate effect size [28] on which to base our sample size because a smaller effect size, if detected, would be unlikely to mandate significant changes in the healthcare system. Allowing for $20 \%$ attrition provides a final sample size of 240. This attrition rate is realistic because of our previous 


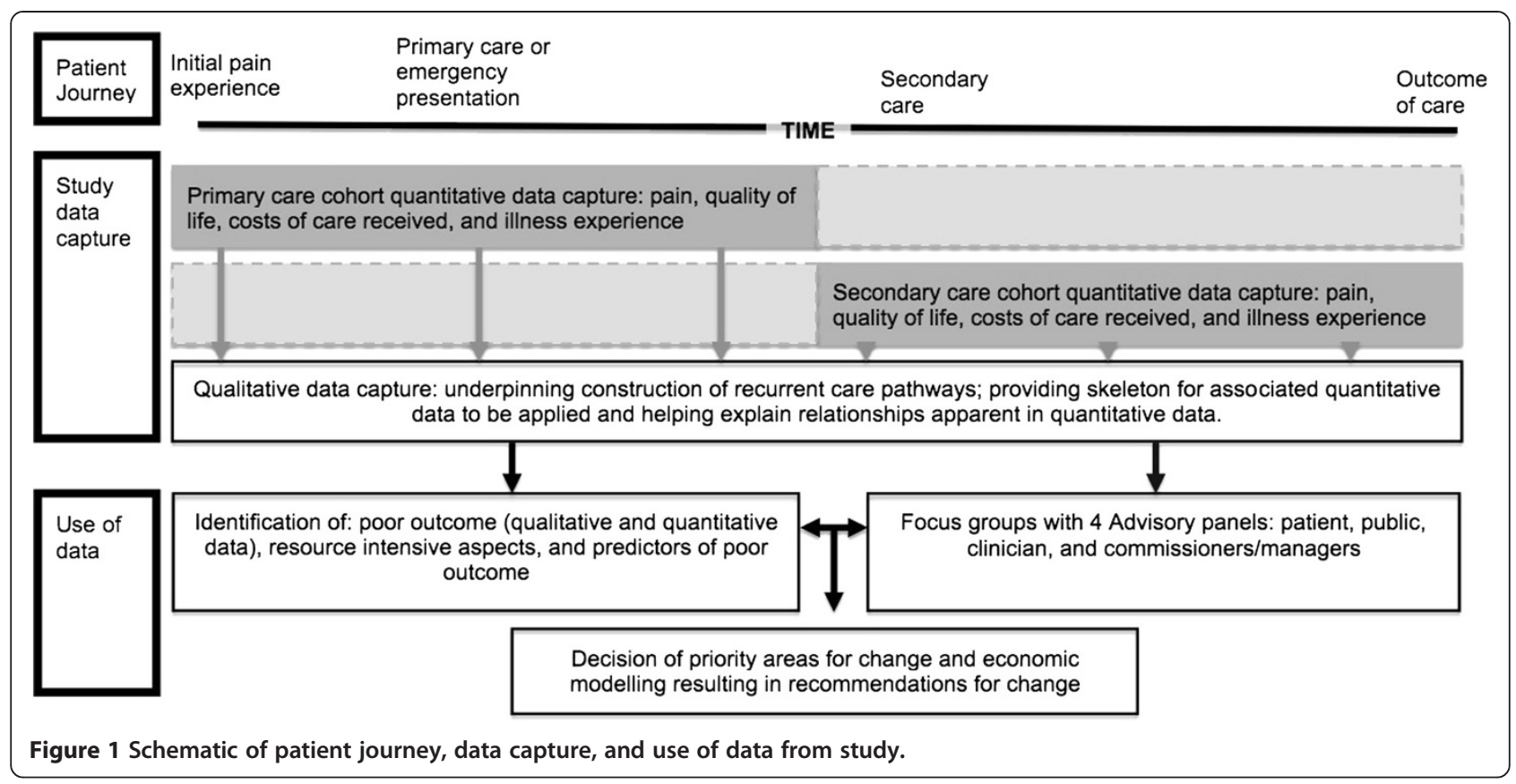

experience with longitudinal data collection with patients suffering from COFP showing a high dropout rate.

\section{Inclusion and exclusion criteria}

Participants will be over the age of eighteen. Orofacial pain will have been present for greater than or equal to three months [29]. Using a validated dual baseline-screening questionnaire (BSQ1) [30,31] the participants will be categorised post-initial recruitment to assign their orofacial pain to a neurological/vascular (from nerves or blood vessels), Dentoalveolar (from tooth or tooth bearing structure), or Temporomandibular disorder/musculoskeletal cause. The sample will be stratified by care sector and gender.

Where a specialist clinical diagnosis is available which suggests a negative screening result from the BSQ1 is a false negative the Chief Investigator (CI) will review the sub-diagnosis automatically generated by the screening questionnaire along with the individual's clinical diagnosis. The CI will then only include the patient in the study if the sub diagnosis or specialist clinical diagnosis is part of the group of conditions being studied: all types of headache, temporomandibular disorders (TMDs), neuralgias, burning mouth syndrome, traumatic neuropathies, and persistent dentoalveolar pain disorder (atypical odontalgia).

The exclusion criteria are that: 1) an individual lacks the capacity to give informed consent for any reason; 2) an individual is categorised by the screening questionnaire as only having dentoalveolar pain, which is not part of the group of conditions that comprise COFP; 3) an individual is unable to communicate complex constructs in English given the qualitative aspects of the overall study.

\section{Recruitment}

The participating clinical and or research team will both prospectively and retrospectively identify individuals eligible to be included in the study on the initial basis of: their age, the duration of their complaint, and presumed diagnosis. Electronic and paper adverts advertising the study will also be placed within the practices participating and any allied clinical facilities such as pharmacies they use. Adverts will also be placed in public places and in the local press if necessary. The advert provides the contact details for the study team and, if appropriate, the individual contacting the study team will be recruited as described below.

For prospective recruitment the research or clinical team will give the patient a short standardised verbal description of the study and ask if the patient is interested in being involved. Standardised recruitment pro forma will be used to record those who are interested in participating in the study and those who decline in order to facilitate an analysis of both those who decline and eventually those who fail to respond or complete the study. Those who are interested in participating will be issued with a phase 1 patient pack, which includes: a patient information sheet, phase 1 consent form and the BSQ1.

For retrospective recruitment standardised letters from the patient's primary care practitioner explaining the study will be sent out to patients seen within the last year with pain fitting the inclusion criteria to ascertain if 


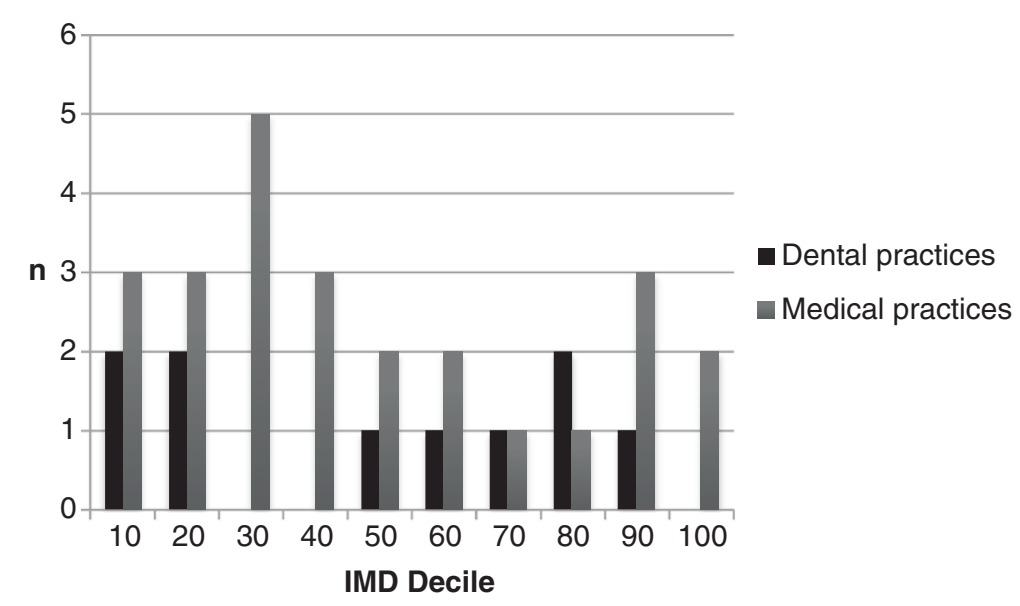

Figure 2 Sociodemographic of practices involved in study. This figure is calculated by using the 2010 UK census data available at http:// neighbourhood.statistics.gov.uk/dissemination/. This census defined 32482 small geographic areas in England each consisting of approximately 1500 people (Lower super-output areas [LSOA]). Each LSOA was assessed and scored according to standardised criteria across 38 domains and then ranked from the best score (rank 1) to the worst (32482). These 38 domains included income, health, and employment [27] and were combined to produce a composite "index of multiple deprivation" (IMD) which was ranked in the same manner. The deciles in the figure above represent the rounded percentage ranking with lower deciles equating to worse deprivation according to IMD score.

they are interested in taking part in the study. Patients contacted in this manner will be asked to contact the research team if they are interested in participating and then the research team will give a standardised verbal explanation of the study by phone, complete the recruitment proforma, and then send out the phase 1 patient pack.

In both prospective and retrospective recruitment, a trained member of the research team will contact those interested within the next fortnight by telephone, or at their next clinic appointment, in order to complete the BSQ1 verbally if the individual is still willing to participate. The BSQ1 is completed by the researcher in accordance with the participant's responses within Excel (Excel v10, Microsoft Corporation, Redmond, WA, USA) and gives an immediate outcome in relation to the inclusion criteria. Those screening positive and giving informed consent, will then be enrolled in the study. Those who withhold consent will be thanked for their interest and will not be enrolled. Those who screen negative will be thanked for their interest and will not be enrolled unless a false negative is suspected whereby the $\mathrm{CI}$ will review all available results for the individual. Those enrolled in the study, after screening positive and giving consent, will be contacted by the research team at a time and location convenient to them to conduct baseline data collection. Any withdrawals from the enrolled cohort will be noted along with age, gender, BSQ1 classification, and the broad reason given for their withdrawal.

\section{Data collection}

All participants $(n=240)$ will complete a baseline structured interview (Case report form [CRF]) with a trained member of the research team, either by phone or face-to-face, in order to: a) capture baseline sociodemographic data; b) capture data about their pain to that point (duration, treatment received and its effectiveness, healthcare practitioners seen); c) ensure their comprehension of the instruments to be used over the next two years.

We have identified the impact of pain, quality of life, and costs as the three most appropriate measures of success in a healthcare system attempting to manage COFP. The instruments used to gather quantitative data on the impact and degree of pain, quality of life, and costs of illness at the varying data collection points (Figure 3 , Table 1 ) will be:

- A quality of life instrument will be issued at each of the six monthly data collection points. This will be the EQ-5D-5 L [24]). At baseline two reference periods will be used for the EQ-5D "last month" and "today" (Questionnaire 1a), thereafter only the standardised reference period of "today" will be used (Questionnaire 1b).

- Multidimensional pain measures - those used will be the Graded Chronic Pain Scale (GCPS) [32] and the West-Haven Yale Multidimensional Pain Inventory (WYMPI version 3) [22]. The baseline questionnaire 2a contains both the GCPS and the Pain impact and Spousal interactions subscales of the WYMPI, but questionnaire $2 \mathrm{~b}$ used sequentially thereafter omits the final subscale of the WYMPI relating to spousal interactions in order to reduce respondent burden.

- Cost of illness instruments - To reduce respondent burden we will issue a "Use of services and 
Initial recruitment prior to screening for eligibility

Patient with three or more months orofacial pain. Verbal description and initial recruitment prior to screening

Complete recruitment proforma and send to team

Follow-up and enrollment

Verbal completion of eligibility Phase 1 Baseline Screening Questionnaire (BSQ1)

Confirm eligibility via BSQ1

Written consent

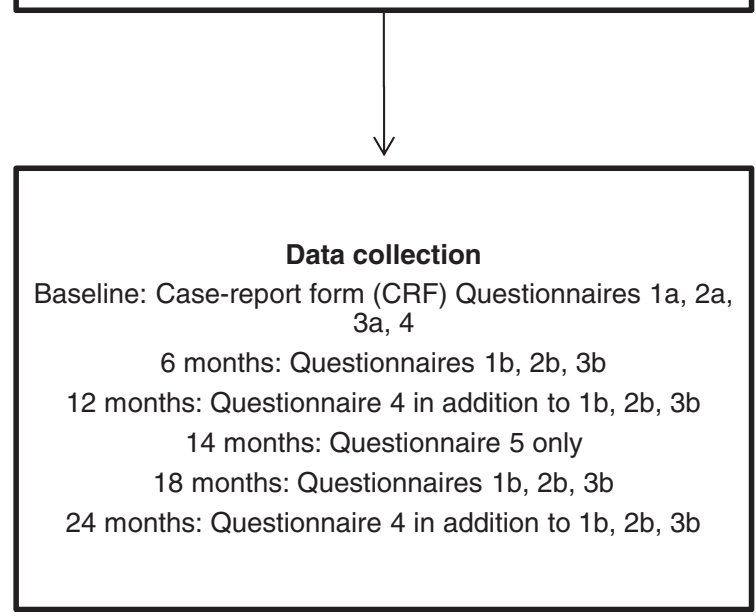

Figure 3 Study flowchart for Phase 1.

productivity" questionnaire at each six monthly data collection points (Questionnaire 3) and a one-off “Time and Travel" questionnaire (Questionnaire 5) [25] at fourteen months into the study. Questionnaire 3 has two versions in order to try and reduce respondent burden: version "a" for administration at baseline, and version "b" for sequential administration thereafter. Version "b" is almost identical to the "a" version other than the omission of some questions that cannot change from baseline for example, "occupation when pain started", and giving options to record sections as "no change" since last administration. Illness perceptions, anxiety and depression will also be briefly examined in order to help profile the study cohort. This will be accomplished through using the IPQ-R and PHQ-4 in questionnaire 4 which will be issued at baseline, twelve and twenty-four months [26,33-36].

A notes-based analysis of consultation, prescription, and referral histories will supplement the self-complete data where necessary and telephone interviews will be held with patients whose data requires further clarification. Throughout the quantitative data collection interim analyses will be conducted on the immature data sets to help identify and explain missing data, and shift the analysis burden.

Any individuals whose data has not been received fourteen days after the instruments were posted to them will be followed up using a standardised operating protocol: reminder letter, then telephone call, and then contact with nominated secondary point of contact if none of the previous modalities of contact have been successful in reaching the individual or their voicemail. An individual will be assumed to have withdrawn in absentia ten days after leaving a message with a voicemail or contacting the secondary point of contact; should they subsequently contact the research team and express a desire to continue all efforts will be made, as far as reasonably practical, to facilitate this.

Qualitative interviews will take place with a purposive sub-sample $(n=30)$ of the total cohort. The sub-sample will be taken using: gender; strata for time in care; type of care environment; origin of COFP (Table 2). Telephone or face-to-face qualitative interviews will be conducted at baseline with this sub-sample by trained and experienced interviewers using a flexible evolving topic guide. It is planned that the same sub-sample will be interviewed again at twelve months and twenty-four months by telephone or face-to-face in order to examine any further experiences or altered perceptions. Data collection and analysis will follow the principles of the constant comparative method [37] whereby data collection and analysis occur concurrently and continue until saturation, which will allow us to add to our sample should interesting issues arise. The interviews aim to build an understanding of: the illness the individual is experiencing, the journey(s) through care, and any apparent relationships in the quantitative data.

\section{Analysis}

Data on use of services (Q3a, 3b and 5) will be used to calculate costs by combining information on resource use with unit costs either developed as study specific estimates or obtained from routinely available sources for example the unit costs of health care [38], the British 
Table 1 Phase 1 questionnaire administration timetable

\begin{tabular}{|c|c|c|c|c|c|c|c|}
\hline \multirow[b]{2}{*}{ Questionnaire } & \multicolumn{7}{|l|}{ Issued at: } \\
\hline & Recruitment & Baseline & $6 \mathrm{M}$ & $12 \mathrm{M}$ & $14 \mathrm{M}$ & $18 \mathrm{M}$ & $24 M$ \\
\hline BSQ1 & $x$ & & & & & & \\
\hline CRF & & $x$ & & & & & \\
\hline EQ-5D (Q1a) & & $x$ & & & & & \\
\hline EQ-5D (Q1b) & & & $x$ & $x$ & & $x$ & $x$ \\
\hline GCPS \& WHYMPI (Q2a) & & $x$ & & & & & \\
\hline GCPS \& WHYMPI (Q2b) & & & $x$ & $x$ & & $x$ & $x$ \\
\hline Baseline use of services and productivity (Q3a) & & $x$ & & & & & \\
\hline Ongoing use of services and productivity (Q3b) & & & $x$ & $x$ & & $x$ & $x$ \\
\hline IPQ-R and PHQ-4 (Q4) & & $x$ & & $x$ & & & $x$ \\
\hline Time and travel questionnaire (Q5) & & & & & $x$ & & \\
\hline
\end{tabular}

National Formulary for medications [39]. The EQ-5D 5 L responses will be converted into health state utilities using tariffs currently under development or cross-walked to the pre-existent EQ-5D-3 L UK population health tariffs [40,41] and will estimate quality adjusted life years (QALYs [42]).

Data from GCPS and WYMPI (Q2a and 2b) will be used in order to build a sequential multidimensional picture of the pain's impact on individuals. As GCPS has been shown to be predictive of outcome in one specific type of COFP (TMDs [23]) it will be examined specifically to see if it provides a good predictor of outcome in primary and or secondary care. The omission of the spousal interactions section from WYMPI after baseline administration will mean that this cannot be examined in relation to change, but it will help categorise our sample at baseline.

The IPQ-R will give us sequential data on the patient's lay perception of their symptoms': identity, cause, severity, consequences, treatment/control. This instrument will also allow us to assess at defined points during their care pathway the emotional impact of their illness as well as their understanding (perceived coherence) of their illness.

Table 2 Purposive subsampling criteria

\begin{tabular}{ll}
\hline Stratification & Details \\
\hline Gender & $\begin{array}{l}\text { Attempt to gain a 1:1 ratio of gender in } \\
\text { subsample }\end{array}$ \\
Care environment & $\begin{array}{l}\text { Attempt to gain a 1:1 ratio of those in primary care } \\
\text { at baseline and those in secondary care at baseline }\end{array}$ \\
Time in care & $\begin{array}{l}\text { Attempt to gain a subsample that contains three } \\
\text { broad groups: }\end{array}$ \\
& First experience of COFP (maximum 6-12 month history) \\
& Moderate experience of COFP (13-23 month history) \\
& Long experience of COFP (>23 month \\
history) & Attempt to get an equal representation of \\
musculoskeletal and neuropathic/vascular origins \\
in sample
\end{tabular}

Scores from the questionnaires are likely to need to be transformed to allow one-way and repeated ANOVA to determine if there are significant differences in cost across quality of life scores and pain. Follow-up regression analysis will then be used to determine the multivariate predictors of these differences including GCPS, care sector, education, and other sociodemographic variables. This data analysis will then help inform the appraisal groups.

\section{Phase two methods: pathway analysis and priority setting}

The aims of Phase 2 are:

1) To develop a model based upon the care pathways reflecting key events (e.g. referrals, use of services, impact on pain and daily living) and use this model to estimate the cost and outcomes (e.g. level of pain, quality of life).

2) To use the data gathered and work with stakeholders, to identify areas where the current pathway might be changed and model the impact of the potential changes on costs, outcomes and cost-effectiveness of care for COFP.

\section{Methods}

The analysis of the qualitative data will be used to assemble the framework (map) of current experiences of the journey through care using an iterative methodology to record recurring emergent experiences against the generic stages of the patients' journeys [6]. The qualitative data will be used to highlight any areas of concern for the sample on the map of the current journey through care with the quantitative data used alongside the qualitative data to help quantify the degree of impact and identify areas that, if changed, may produce the most benefit to the patients and health service. This will be an iterative process that will produce an understanding and inform a 
model that will be developed to describe the care pathways experienced.

The map and quantitative longitudinal data will in themselves illustrate areas of high cost and poor outcome (pain, quality of life) but the discussion on which areas of the pathway are a priority to change will be conducted with four separate appraisal groups: public, patient, clinician, and commissioning and managing groups, each consisting of 5-8 members of a variety of ages from the local area. COFP patients and the public (lay) participants will be recruited from routine diagnostic clinics in the Newcastle-Upon-Tyne Hospitals Trust using a standardised PIS and consent form. The inclusion and exclusion criteria for patients will mirror that of phase 1 using the same screening questionnaire. To be accepted as a member of the public the following inclusion criteria must be met through responding to a short screening questionnaire:

- The individual in question nor their family have on-going pain in their mouth and or face within the last twelve months

- The individual in question nor their family are health professionals

A standardised letter will be used to approach local clinicians and service managers/commissioners at their respective professional addresses. The letter will contain the Patient Information Sheet (PIS) and consent form and contact details for the research team.

The appraisal groups will be presented with the study findings in a comprehensible annonymised form two weeks prior to convening and upon convening will be conducted in a focus group manner. The groups will be recorded and transcribed verbatim, helping guide the decisions on the priority areas in the current care pathway to change in the subsequent economic modelling. Trained facilitators using flexible and evolving topic guides will undertake the focus groups. The groups will be digitally recorded and transcribed verbatim. Qualitative analysis of the focus group data will follow the same principles as those described in Phase 1.

\section{Phase three methods: economic modelling and recommendations}

The aim of phase three is:

1) Using the results of phase one and two, develop recommendations for practice and future research This phase consists of developing an economic model based upon the care pathways determined in phases 1 and 2. The model will describe the logical and temporal sequence of events from first presentation with COFP in primary care through any subsequent management in both primary and secondary care. The model will be developed in line with best practice [43]. We anticipate that the model may take the form of a Markov model but the precise form of the model will be determined as part of the project and will be chosen to fit the processes modelled. The outputs of the model will be cumulative costs and QALYs over a 5-year period (i.e. the time period over which we believe data can reliably extrapolated) but we will explore in a sensitivity analysis the impact of conducting a longer (e.g. lifetime) time horizons. The perspective will be that of the UK NHS and patients and discounting in the base case will be at 3.5\% [44].

The parameter estimates (probabilities, costs and utilities) required for the model will come from the longitudinal study described above, focused searches of the literature and advice from an expert panel. All uncertainty surrounding estimates of input parameters will be informed by appropriate distributions calculated from the longitudinal study or the literature. The results of the economic model will be presented as incremental costs and QALYs, and the incremental cost per QALY gained. Both deterministic and probabilistic sensitivity analyses will be carried out to test and explore uncertainties. The results of the probabilistic sensitivity analysis will be presented as a series of cost-effectiveness acceptability curves (CEACs). At the end of the modelling process recommendations for service change will be available for evaluation and further research.

\section{Ethical approval}

This study has approval from the Yorkshire and the Humber (Leeds West) Ethics Committee (Ref: 12/YH/0338) and NHS R\&D approval from each participating site. Honorary NHS contracts have been issued where necessary.

\section{Discussion}

Currently there are data, which suggest that patients with COFP use a large amount of healthcare resource [13-17]. What is unknown, and this study seeks to identify, is if the resource that is used is proportional and effective for their complaint?

It is conceivable that the only reason COFP patients use so much resource is because the care system they experience fails to provide them with clear and defined pathways of care based upon early diagnosis and appropriate management. The need for early diagnosis and management is key given that those with a propensity for developing psychological comorbidities may develop these 
sooner if diagnosis is delayed or there is a misdiagnosis causing uncertainty or anxiety over the nature of the complaint $[5,45,46]$. Any psychological comorbidities that develop will then negatively impact on their prognosis [23,47-49].

Early appropriate conservative management [50] is also important given the emerging role for central sensitisation [51-57] and the autonomic nervous system (ANS) in COFP [58]. Reducing the peripheral afferent barrage at the earliest opportunity and down regulating any dysfunctional ANS as soon as possible through early diagnosis, reassurance, and management will also all hopefully reduce the chance of central neuroplastic changes, "central sensitisation" $[51,59]$. Reducing the potential for central sensitisation, or up regulation of the ANS, occurring may then help improve the success of (simpler) therapies, reduce treatment times, and improve prognosis by reducing the potential for the condition becoming chronic.

There are clear and evidence-based methods for managing generic chronic pain in an interdisciplinary fashion of which a substantial proportion may be translatable into COFP $[60,61]$. A recent national pain audit [21] in the U.K. has, however, highlighted the difficulty in establishing the provision of such a service for generic chronic pain management nationwide, irrespective of the need for a condition specific service. The same audit also highlighted that despite the evidence base supporting interdisciplinary pain management, it is still unclear how to clinically and cost effectively provide (NHS) healthcare services for patients with chronic pain [21]. Against the background of changes in commissioning and the drive to provide services more cost-effectively in the NHS [62] the DEEP study may provide answers for COFP care pathways and also a model and methodology by which to examine other long-term conditions' care pathways.

\section{Abbreviations \\ ANS: Autonomic nervous system; ANOVA: Analysis of variance; BSQ1: Baseline screening questionnaire completed in phase 1 prior to enrolment; Cl: Chief investigator; CEAC: Cost effectiveness acceptability curve; COFP: Chronic orofacial pain; CRF: Case report form; DEEP: Developing Effective and Efficient care Pathways in chronic pain; GCPS: Graded Chronic Pain Scale; IPQ- R: Revised illness perceptions questionnaire; NHS: National Health Service in UK; PHQ-4: Patient health questionnaire 4; PIS: Patient information sheet; QALYs: Quality adjusted life years; TMDs: Temporomandibular disorders; WYHMPI: West-haven Yale Multidimensional Pain inventory.}

\section{Competing interests}

The authors declare that they have no competing interests.

\section{Authors' contributions}

JD, MB, CE, JGS, VAS, LV were all involved in original conception and design of the study. All authors helped draft this manuscript and were involved in revising the manuscript critically for important intellectual content and have given approval of the final manuscript.

\section{Authors' information}

JD is a Senior Lecturer in Oral Surgery and Orofacial Pain and NIHR Clinician Scientist. MB is a Research Associate. VAS is a Senior Lecturer in Health
Psychology. CE is a Senior Lecturer in Medical Sociology. JGS is a Professor of Oral Health Services Research. LV is a Professor of Health Economics.

\section{Acknowledgements}

This study and JD are funded by a NIHR Clinician Scientist award (NIHR-CS-011-003). The views expressed in this publication are those of the author(s) and not necessarily those of the NHS, the National Institute for Health Research, or the Department of Health in the United Kingdom.

Received: 17 December 2013 Accepted: 13 January 2014 Published: 21 January 2014

\section{References}

1. Aggarwal VR, Joughin A, Zakrzewska J, Appelbe P, Tickle M: Dentist's preferences for diagnosis, management and referral of chronic oro-facial pain: results from a national survey. Health Educ J 2012, 71:662-669.

2. Aggarwal VR, Joughin A, Zakrzewska JM, Crawford FJ, Tickle M: Dentists' and specialists' knowledge of chronic orofacial pain: results from a continuing professional development survey. Prim Dent Care 2011, 18:41-44.

3. Durham J, Exley C, Wassell R, Steele JG: 'Management is a black art'-professional ideologies with respect to temporomandibular disorders. Br Dent J 2007, 202:E29. discussion 682-3.

4. Kristiansson $\mathrm{MH}$, Brorsson A, Wachtler C, Troein M: Pain, power and patience - a narrative study of general practitioners' relations with chronic pain patients. BMC Fam Pract 2011, 12:31.

5. Durham J, Steele JG, Wassell RW, Exley C: Living with uncertainty: temporomandibular disorders. J Dent Res 2010, 89:827-830.

6. Durham J, Steele J, Moufti MA, Wassell R, Robinson P, Exley C: Temporomandibular disorder patients' journey through care. Community Dent Oral Epidemiol 2011, 39:532-541.

7. Nilsson IM, List T, Willman A: Adolescents with temporomandibular disorder pain-the living with TMD pain phenomenon. J Orofac Pain 2011, 25:107-116.

8. Wolf E, Birgerstam P, Nilner M, Petersson K: Nonspecific chronic orofacial pain: studying patient experiences and perspectives with a qualitative approach. J Orofac Pain 2008, 22:349-358.

9. Wolf $E$, Birgerstam P, Nilner M, Petersson K: Patients' experiences of consultations for nonspecific chronic orofacial pain: a phenomenological study. J Orofac Pain 2006, 20:226-233.

10. Hasanain F, Durham J, Moufti A, Steen IN, Wassell RW: Adapting the diagnostic definitions of the RDC/TMD to routine clinical practice: a feasibility study. J Dent 2009, 37:955-962.

11. Meloto CB, Serrano PO, Ribeiro-DaSilva MC, Rizzatti-Barbosa CM: Genomics and the new perspectives for temporomandibular disorders. Arch Oral Biol 2011, 56:1181-1191.

12. Beecroft EV, Durham J, Thomson P: Retrospective examination of the healthcare 'journey' of chronic orofacial pain patients referred to oral and maxillofacial surgery. Br Dent $J$ 2013, 214:E12.

13. Aggarwal VR, McBeth J, Zakrzewska JM, Macfarlane GJ: Unexplained orofacial pain - is an early diagnosis possible? Br Dent J 2008, 205:E6. discussion 140-1.

14. Glaros AG, Glass EG, Hayden WJ: History of treatment received by patients with TMD: a preliminary investigation. J Orofac Pain 1995, 9:147-151.

15. Shimshak DG, Kent RL, DeFuria M: Medical claims profiles of subjects with temporomandibular joint disorders. Cranio 1997, 15:150-158.

16. Shimshak DG, DeFuria MC: Health care utilization by patients with temporomandibular joint disorders. Cranio 1998, 16:185-193.

17. White BA, Williams LA, Leben JR: Health care utilization and cost among health maintenance organization members with temporomandibular disorders. J Orofac Pain 2001, 15:158-169.

18. Suvinen TI, Reade PC, Kemppainen P, Kononen M, Dworkin SF: Review of aetiological concepts of temporomandibular pain disorders: towards a biopsychosocial model for integration of physical disorder factors with psychological and psychosocial illness impact factors. Eur J Pain 2005, 9:613-633.

19. Phillips C, Main C, Buck R, Aylward M, Wynne-Jones G, Farr A: Prioritising pain in policy making: the need for a whole systems perspective. Health Policy 2008, 88:166-175.

20. Donaldson L: Pain: Breaking through the Barrier. In 150 years of the Annual Report of the Chief Medical Officer: On the state of Public Health. 2008:32-39. 
21. National pain audit. Final report 2010-2012. [http://www.nationalpainaudit. org/media/files/NationalPainAudit-2012.pdf].

22. Kerns RD, Turk DC, Rudy TE: The West Haven-Yale Multidimensional Pain Inventory (WHYMPI). Pain 1985, 23:345-356.

23. Von Korff M, Dunn KM: Chronic pain reconsidered. Pain 2008, 138:267-276.

24. Herdman M, Gudex C, Lloyd A, Janssen M, Kind P, Parkin D, Bonsel G, Badia X: Development and preliminary testing of the new five-level version of EQ-5D (EQ-5D-5 L). Qual Life Res 2011, 20:1727-1736.

25. Wordsworth S, Thompson S: An annotated cost questionnaire for patients: results of piloting. HERU Dicussion Paper 2001, 03/01.

26. Moss-Morris R, Weinman J, Petrie KJ, Horne R, Cameron LD, Buick D: The revised illness perception questionnaire (IPQ-R). Psychol Health 2002, 17:1-16.

27. English indices of deprivation 2010. [https://www.gov.uk/government/ uploads/system/uploads/attachment_data/file/6871/1871208.pdf].

28. Cohen J: Statistical Power Analysis for the Behavioral Sciences (2nd ed). New Jersey: Lawrence Erlbaum; 1988.

29. IASP classification of chronic pain: introduction. Pain 1986, 24(Supplement 1):S3-S8.

30. Gonzalez YM, Schiffman E, Gordon SM, Seago B, Truelove EL, Slade G, Ohrbach R: Development of a brief and effective temporomandibular disorder pain screening questionnaire: reliability and validity. J Am Dent Assoc 2011, 142:1183-1191.

31. Hapak L, Gordon A, Locker D, Shandling M, Mock D, Tenenbaum HC Differentiation between musculoligamentous, dentoalveolar, and neurologically based craniofacial pain with a diagnostic questionnaire. J Orofac Pain 1994, 8:357-368.

32. Smith BH, Penny Kl, Purves AM, Munro C, Wilson B, Grimshaw J, Chambers WA, Smith WC: The chronic pain grade questionnaire: validation and reliability in postal research. Pain 1997, 71:141-147.

33. Sniehotta FF, Gorski C, Araujo-Soares V: Adoption of community-based cardiac rehabilitation programs and physical activity following phase III cardiac rehabilitation in Scotland: a prospective and predictive study. Psychol Health 2010, 25:839-854.

34. Kroenke K, Spitzer RL, Williams JB, Monahan PO, Lowe B: Anxiety disorders in primary care: prevalence, impairment, comorbidity, and detection. Ann Intern Med 2007, 146:317-325.

35. Kroenke K, Spitzer RL, Williams JB, Lowe B: An ultra-brief screening scale for anxiety and depression: the PHQ-4. Psychosomatics 2009, 50:613-621.

36. Lowe B, Wahl I, Rose M, Spitzer C, Glaesmer H, Wingenfeld K, Schneider A, Brahler E: A 4-item measure of depression and anxiety: validation and standardization of the patient health questionnaire-4 (PHQ-4) in the general population. J Affect Disord 2010, 122:86-95.

37. Glaser BG: The constant comparative method of qualitative analysis. Soc Probl 1965, 12:436-445.

38. Curtis L: Unit Costs of Health and Social Care. Kent: Personal Social Services Research Unit; 2012 [http://www.pssru.ac.uk/project-pages/unit-costs/].

39. British national formulary. [http://www.bnf.org/bnf/index.htm].

40. van Hout B, Janssen MF, Feng YS, Kohlmann T, Busschbach J, Golicki D, Lloyd A, Scalone L, Kind P, Pickard AS: Interim scoring for the EQ-5D-5 L: mapping the EQ-5D-5 L to EQ-5D-3 L value sets. Value Health 2012, 15:708-715.

41. Kind P, Dolan P, Gudex C, Williams A: Variations in population health status: results from a United Kingdom national questionnaire survey. BMJ 1998, 316:736-741.

42. The role of the EuroQOL instrument in QALY calculations. [http://www.york.ac.uk/media/che/documents/papers/discussionpapers/CHE \%20Discussion\%20Paper\%20130.pdf].

43. Philips Z, Ginnelly L, Sculpher M, Claxton K, Golder S, Riemsma R, Woolacoot N, Glanville J: Review of guidelines for good practice in decision-analytic modelling in health technology assessment. Health Technol Assess 2004, 8:1-158, iii-iv, ix-xi.

44. Guide to the methods of technology appraisal. [http://publications.nice. org.uk/guide-to-the-methods-of-technology-appraisal-2013-pmg9].

45. Durham J, Exley C, John MT, Nixdorf DR: Persistent dentoalveolar pain: the patient's experience. J Orofac Pain 2013, 27:6-13.

46. Garro LC: Narrative representations of chronic illness experience: cultural models of illness, mind, and body in stories concerning the temporomandibular joint (TMJ). Soc Sci Med 1994, 38:775-788.

47. Epker J, Gatchel RJ, Ellis E: A model for predicting chronic TMD: practical application in clinical settings. J Am Dent Assoc 1999, 130:1470-1475.
48. Garofalo JP, Gatchel RJ, Wesley AL, Ellis E 3rd: Predicting chronicity in acute temporomandibular joint disorders using the research diagnostic criteria. J Am Dent Assoc 1998, 129:438-447.

49. Wright AR, Gatchel RJ, Wildenstein L, Riggs R, Buschang P, Ellis E: Biopsychosocial differences between high-risk and low-risk patients with acute TMD-related pain. J Am Dent Assoc 2004, 135:474-483.

50. Greene CS: Managing the care of patients with temporomandibular disorders: a new guideline for care. J Am Dent Assoc 2010, 141:1086-1088.

51. Woolf $\mathrm{CJ}$ : Central sensitization: implications for the diagnosis and treatment of pain. Pain 2011, 152:S2-15.

52. Anderson GC, John MT, Ohrbach R, Nixdorf DR, Schiffman EL, Truelove ES, List T: Influence of headache frequency on clinical signs and symptoms of TMD in subjects with temple headache and TMD pain. Pain 2011, 152:765-771.

53. Cady RJ, Glenn JR, Smith KM, Durham PL: Calcitonin gene-related peptide promotes cellular changes in trigeminal neurons and glia implicated in peripheral and central sensitization. Mol Pain 2011, 7:94

54. Fernandez-de-las-Penas C, Galan-del-Rio F, Fernandez-Carnero J, Pesquera J, Arendt-Nielsen L, Svensson P: Bilateral widespread mechanical pain sensitivity in women with myofascial temporomandibular disorder: evidence of impairment in central nociceptive processing. J Pain 2009, 10:1170-1178.

55. Lorduy KM, Liegey-Dougall A, Haggard R, Sanders CN, Gatchel RJ: The prevalence of comorbid symptoms of central sensitization syndrome among three different groups of temporomandibular disorder patients. Pain Pract 2013, 2013:604-613.

56. Mohn C, Vassend O, Knardahl S: Experimental pain sensitivity in women with temporomandibular disorders and pain-free controls: the relationship to orofacial muscular contraction and cardiovascular responses. Clin J Pain 2008, 24:343-352.

57. Xu W, Wu Y, Bi Y, Tan L, Gan Y, Wang K: Activation of voltage-gated $\mathrm{KCNQ} / \mathrm{Kv} 7$ channels by anticonvulsant retigabine attenuates mechanical allodynia of inflammatory temporomandibular joint in rats. Mol Pain 2010, 6:49.

58. Maixner W, Greenspan JD, Dubner R, Bair E, Mulkey F, Miller V, Knott C, Slade GD, Ohrbach R, Diatchenko L, Fillingim RB: Potential autonomic risk factors for chronic TMD: descriptive data and empirically identified domains from the OPPERA case-control study. J Pain 2011, 12:T75-91.

59. Dodick D, Silberstein S: Central sensitization theory of migraine: clinical implications. Headache 2006, 46(Suppl 4):S182-91.

60. Gardea MA, Gatchel RJ: Interdisciplinary treatment of chronic pain. Curr Rev Pain 2000, 4:18-23.

61. Robbins H, Gatchel RJ, Noe C, Gajraj N, Polatin P, Deschner M, Vakharia A, Adams $L:$ A prospective one-year outcome study of interdisciplinary chronic pain management: compromising its efficacy by managed care policies. Anesth Analg Anesth Analg 2003, 97:156-162.

62. Equity and excellence: liberating the NHS. [http://www.dh.gov.uk/ prod_consum_dh/groups/dh_digitalassets/@dh/@en/@ps/documents/ digitalasset/dh_117794.pdf].

doi:10.1186/1472-6831-14-6

Cite this article as: Durham et al:: Developing Effective and Efficient care pathways in chronic Pain: DEEP study protocol. BMC Oral Health 2014 14:6.

\section{Submit your next manuscript to BioMed Central and take full advantage of:}

- Convenient online submission

- Thorough peer review

- No space constraints or color figure charges

- Immediate publication on acceptance

- Inclusion in PubMed, CAS, Scopus and Google Scholar

- Research which is freely available for redistribution 